\title{
FOLFIRINOX-induced reversible dysarthria: A case report and review of previous cases
}

\author{
AYUMU MATSUOKA ${ }^{1,2}$, OSAMU MAEDA ${ }^{1}$, MEGUMI INADA-INOUE $^{1}$, EIZABURO OHNO ${ }^{3}$, \\ YOSHIKI HIROOKA ${ }^{3}$, YUKIHIRO YOKOYAMA ${ }^{4}$, TSUTOMU FUJII ${ }^{5}$, \\ MASATO NAGINO ${ }^{4}$, HIDEMI GOTO ${ }^{2,3}$ and YUICHI ANDO $^{1}$ \\ ${ }^{1}$ Department of Clinical Oncology and Chemotherapy, Nagoya University Hospital; \\ ${ }^{2}$ Department of Gastroenterology and Hepatology, Nagoya University Graduate School of Medicine; \\ ${ }^{3}$ Department of Endoscopy, Nagoya University Hospital; ${ }^{4}$ Division of Surgical Oncology, Department of Surgery, \\ Nagoya University Graduate School of Medicine; ${ }^{5}$ Department of Gastroenterological Surgery (Surgery II), \\ Nagoya University Graduate School of Medicine, Nagoya, Aichi 466-8550, Japan
}

Received October 4, 2014; Accepted July 17, 2015

DOI: $10.3892 / 01.2015 .3591$

\begin{abstract}
FOLFIRINOX is a standard chemotherapeutic regimen for patients with advanced pancreatic cancer who have a good performance status. In this study, we present the case of a 64-year-old male who developed dysarthria following FOLFIRINOX treatment, and review all four cases of dysarthria encountered among the nine patients who received this treatment in our hospital. In all cases, dysarthria occurred during the infusion of irinotecan in the first course of treatment, persisted for several hours, and then resolved rapidly without any sequelae. Physical and neurological examinations at the onset of dysarthria revealed no other abnormalities. Imaging studies revealed no abnormal findings. Atropine was prophylactically administered in the second and subsequent courses of treatment and effectively prevented or alleviated dysarthria. This acute neurological symptom is surprising and uncommon in traditional cancer chemotherapy, and medical oncologists may initially suspect the onset of stroke or cerebrovascular disease. However, consistent with our experience, all reported cases resolved completely, with no need for dose reduction or treatment interruption.
\end{abstract}

\section{Introduction}

FOLFIRINOX, a combination chemotherapy regimen consisting of fluorouracil, leucovorin, irinotecan and oxaliplatin, is currently a standard treatment for patients with advanced

Correspondence to: Dr Ayumu Matsuoka, Department of Clinical Oncology and Chemotherapy, Nagoya University Hospital, 65 Tsuruma-Cho, Showa-Ku, Nagoya, Aichi 466-8550, Japan E-mail: ayumu0611@med.nagoya-u.ac.jp

Key words: FOLFIRINOX, dysarthria, irinotecan, oxaliplatin, pancreatic cancer pancreatic cancer who have a good performance status (1). However, FOLFIRINOX can cause severe toxicities, including neutropenia, febrile neutropenia, thrombocytopenia, fatigue and diarrhea, frequently requiring dose reduction or treatment interruption. Irinotecan itself rarely causes dysarthria, which is considered to be a type of acute cholinergic syndrome $(2,3)$. Due to the notable discordance in the incidence of dysarthria between FOLFIRINOX and other irinotecan-containing regimens, we speculate that FOLFIRINOX-induced dysarthria is associated with the sequence of drug administration in this regimen (i.e., intravenous infusion of oxaliplatin, immediately followed by irinotecan). Since oxaliplatin is infused before irinotecan in FOLFIRINOX, oxaliplatin exaggerates the cholinergic effects of irinotecan, making dysarthria increasingly evident.

In the present study we report a case of transient dysarthria, a speech disorder caused by disturbances of the muscles involved in speech, which occurred during the intravenous infusion of irinotecan as part of a FOLFIRINOX regimen in a 64-year-old male patient. We also review other cases previously observed in our hospital. The study was approved by the ethics committee of Nagoya University Hospital (Nagoya, Japan; approval no. 2014-0151).

\section{Case report}

A 64-year-old Japanese male was referred to Nagoya University Hospital due to metastatic pancreatic cancer. The patient had a history of diabetes mellitus and was receiving an oral DPP4 inhibitor (Vildagliptin). He had no history of allergy or adverse reactions to specific drugs. He received FOLFIRINOX as first-line chemotherapy, which consisted of oxaliplatin $85 \mathrm{mg} / \mathrm{m}^{2}$ administered intravenously over the course of $2 \mathrm{~h}$, followed by irinotecan $180 \mathrm{mg} / \mathrm{m}^{2}$ over the course of $90 \mathrm{~min}$ and $l$-leucovorin $200 \mathrm{mg} / \mathrm{m}^{2}$ over the course of $2 \mathrm{~h}$, immediately followed by fluorouracil $400 \mathrm{mg} / \mathrm{m}^{2}$ as an intravenous bolus and then $2,400 \mathrm{mg} / \mathrm{m}^{2}$ as a $46-\mathrm{h}$ continuous infusion, usually with anti-emetic premedication with 
palonosetron and dexamethasone. Prophylactic atropine was not used in the first course. The patient's complete blood count and biochemical test results before the start of chemotherapy were within normal limits. During the first course of FOLFIRINOX, dysarthria developed 90 min after starting the irinotecan infusion and was accompanied by rhinitis, diaphoresis, acute-onset diarrhea and abdominal pain, which are typical signs and symptoms of acute cholinergic syndrome, persisting for $\sim 2 \mathrm{~h}$. These symptoms were alleviated by intramuscular atropine. The patient was conscious and alert, and physical and neurological examinations at the onset of dysarthria revealed no apparent abnormalities. In the second and subsequent courses of chemotherapy, prophylactic treatment with atropine was effective, and the patient did not suffer dysarthria or any other cholinergic symptoms.

Since the approval of FOLFIRINOX for pancreatic cancer in Japan in December 2013, four cases of dysarthria have been encountered among nine patients who received FOLFIRINOX in our hospital (Table I). In all cases, dysarthria occurred during the infusion of irinotecan in the first course of treatment and then resolved rapidly without any sequelae. Certain patients experienced distal extremity paresthesia and pharyngolaryngeal dysesthesia, which are known manifestations of oxaliplatin-induced acute neurotoxicity. All patients remained conscious and alert, and physical and neurological examinations at the onset of dysarthria revealed no other abnormalities. Imaging studies, including computed tomography and magnetic resonance imaging of the brain, were performed on Patient 1, and revealed no abnormalities. Atropine effectively palliated the symptoms at the onset of dysarthria, as well as prophylactically in the subsequent courses.

\section{Discussion}

Two significant clinical lessons have been learned from our experience with these patients. First, FOLFIRINOX frequently causes transient dysarthria. Second, all cases resolved completely, with no need for dose reduction or treatment interruption.

With regard to the first point, since the approval of this regimen in Japan, it has been noted that FOLFIRINOX causes reversible dysarthria more often than previously reported for irinotecan monotherapy and other irinotecan-containing regimens (e.g., FOLFIRI and FOLFOXIRI) (3-12). In our hospital, overt speech disturbance diagnosed as dysarthria developed in 4 of the 9 patients (44.4\%) who received FOLFIRINOX. Although dysarthria was not clearly recognized in the original ACCORD11 trial (1), a substantial number of patients developed this symptom in subsequent studies; Gunturu et al (4) reported nine cases of dysarthria among 35 patients (25.7\%), and a phase II trial in Japan reported five cases among 36 patients (13.8\%) (5). Therefore, the unexpectedly high incidence of dysarthria appears to be a characteristic adverse effect of FOLFIRINOX that extends beyond ethnicity. Conversely, it is known that irinotecan directly causes dysarthria, although only 10 cases have been reported in the literature (2,3,6-13). Although irinotecan exerts its antitumor activity after being metabolized to $\mathrm{SN}-38$ in vivo, dysarthria is apparently caused by the parent compound irinotecan binding to the active site of 
acetylcholinesterase and eliciting a type of acute cholinergic syndrome (14). The hypoglossal nerve, which plays a major role in speech function through its innervation of tongue muscles, has increased intrinsic sensitivity to cholinergic stimulation owing to the higher density of cholinergic receptors compared with other brainstem nuclei $(15,16)$, which would explain the cause of dysarthria. Other cholinergic symptoms, including rhinitis, diaphoresis and intestinal hyperperistalsis, occasionally occur simultaneously with dysarthria, and atropine effectively palliates the symptoms of dysarthria, supporting the notion that dysarthria is caused by increased acetylcholine activity. Due to the significant discordance in the incidence of dysarthria between FOLFIRINOX and other irinotecan-containing regimens, we speculate that FOLFIRINOX-induced dysarthria is associated with the sequence of drug administration (i.e., intravenous infusion of oxaliplatin, immediately followed by irinotecan). No cases of dysarthria were reported in clinical trials of FOLFOXIRI in advanced colorectal cancer (in which irinotecan was administered prior to oxaliplatin) (17-19). Oxaliplatin frequently causes acute peripheral neurotoxicity, characterized by transient, cold-induced distal and perioral paresthesias and pharyngolaryngeal dysesthesias, attributed to hyperexcitability of peripheral nerves during or immediately following infusion (20). Since oxaliplatin is infused prior to irinotecan in FOLFIRINOX, the hypoglossal nerve stimulation caused by initial treatment with oxaliplatin exaggerates the cholinergic effects of irinotecan, making dysarthria increasingly evident.

The second point was that all reported cases resolved completely, with no need for dose reduction or treatment interruption. As the sudden occurrence of dysarthria is a surprising and uncommon adverse event in traditional cancer chemotherapy, medical oncologists may initially suspect an acute onset of stroke or cerebrovascular disease. However, consistent with our experience, all reported cases were completely reversible, and neither dose reduction nor treatment interruption was necessary.

In conclusion, FOLFIRINOX frequently causes dysarthria, which is transient and resolves spontaneously without any sequelae. Medical oncologists need to correctly identify this characteristic adverse effect of FOLFIRINOX in order to avoid unnecessary dose reduction or treatment interruption.

\section{References}

1. Conroy T, Desseigne F, Ychou M, Bouché O, Guimbaud R, Bécouarn Y, Adenis A, Raoul JL, Gourgou-Bourgade S, de la Fouchardière $\mathrm{C}$, et al: FOLFIRINOX versus gemcitabine for metastatic pancreatic cancer. N Engl J Med 364: 1817-1825, 2011.

2. Hamberg P, De Jong FA, Brandsma D, Verweij J and Sleijfer S: Irinotecan-induced central nervous system toxicity. Report on two cases and review of the literature. Acta Oncol 47: 974-978, 2008 .

3. Sogabe S, Yuki S, Takano H, Kobayashi Y, Nakatsumi H, Sasaki T, Kawamoto Y, Fukushima H, Iwanaga I, Uehata Y, et al: A case of sigmoid colon cancer with temporary dysarthria associated with irinotecan. Gan To Kagaku Ryoho 38: 1375-1377, 2011 (In Japanese).
4. Gunturu KS, Yao X, Cong X, Thumar JR, Hochster HS, Stein SM and Lacy J: FOLFIRINOX for locally advanced and metastatic pancreatic cancer: single institution retrospective review of efficacy and toxicity. Med Oncol 30: 361, 2013.

5. Okusaka T, Ikeda M, Fukutomi A, Ioka T, Furuse J, Ohkawa S, Isayama $\mathrm{H}$ and Boku $\mathrm{N}$ : Phase II study of FOLFIRINOX for chemotherapy-naïve Japanese patients with metastatic pancreatic cancer. Cancer Sci 105: 1321-1326, 2014.

6. Lee KA, Kang HW, Ahn JH, Suk HJ and Kim H: Dysarthria induced by irinotecan in a patient with colorectal cancer. Am J Health Syst Pharm 70: 1140-1143, 2013.

7. Sevilla Garcia I, Rueda A and Alba E: Irinotecan-induced central nervous system toxicity: a case report. J Natl Cancer Inst 91: 647, 1999 .

8. Baz DV, Bofill JS and Nogueira JA: Irinotecan-induced dysarthria. J Natl Cancer Inst 93: 1419-1420, 2001.

9. Ceccaldi B, Kara F, Mommeja-Marin $H$, Bègue $M$, Saint Blancard P, Le Marec E and Hauteville D: Dysarthria during irinotecan administration. Rev Med Interne 23: 950-951, 2002 (In French).

10. De Marco S, Squilloni E, Vigna L, Bertagnolio MF and Sternberg CN: Irinotecan chemotherapy associated with transient dysarthria and aphasia. Ann Oncol 15: 1147-1148, 2004.

11. Hamberg P, Donders RC and Ten Bokkel Huinink D: Central nervous system toxicity induced by irinotecan. J Natl Cancer Inst 98: 219, 2006.

12. Gomez JA, Sanchez I and Ramirez JA: Irinotecan-induced dysarthria: an insight into its pathogenesis? Gastrointest Cancer Res 2: 209-210, 2008.

13. Dressel AJ, van der Mijn JC, Aalders IJ, Rinkel RN and van der Vliet HJ: Irinotecan-induced dysarthria. Case Rep Oncol 5: 47-51, 2012.

14. Harel M, Hyatt JL, Brumshtein B, Morton CL, Yoon KJ, Wadkins RM, Silman I, Sussman JL and Potter PM: The crystal structure of the complex of the anticancer prodrug 7-ethyl10-[4-(1-piperidino)-1piperidino]-carbonyloxycamptothecin (CPT-11) with Torpedo californica acetylcholinesterase provides a molecular explanation for its cholinergic action. Mol Pharmacol 67: 1874-1881, 2005.

15. Haxhiu MA, Mitra J, van Lunteren E, Bruce EN and Cherniack NS: Hypoglossal and phrenic responses to cholinergic agents applied to ventral medullary surface. Am J Physiol 247: R939-R944, 1984.

16. Cortes R, Probst A and Palacios JM: Quantitative light microscopic autoradiographic localization of cholinergic muscarinic receptors in the human brain: brainstem. Neuroscience 12: 1003-1026, 1984.

17. Falcone A, Ricci S, Brunetti I, Pfanner E, Allegrini G, Barbara C, Crinò L, Benedetti G, Evangelista W, Fanchini L, et al: Phase III trial of infusional fluorouracil, leucovorin, oxaliplatin and irinotecan (FOLFOXIRI) compared with infusional fluorouracil, leucovorin and irinotecan (FOLFIRI) as first-line treatment for metastatic colorectal cancer: The Gruppo Oncologico Nord Ovest. J Clin Oncol 25: 1670-1676, 2007.

18. Souglakos J, Androulakis N, Syrigos K, Polyzos A, Ziras N, Athanasiadis A, Kakolyris S, Tsousis S, Kouroussis Ch, Vamvakas L, et al: FOLFOXIRI (folinic acid, 5-fluorouracil, oxaliplatin and irinotecan) vs. FOLFIRI (folinic acid, 5-fluorouracil and irinotecan) as first-line treatment in metastatic colorectal cancer (MCC): a multicenter randomized phase III trial from the Hellenic oncology research group (HORG). Br J Cancer 94: 798-805, 2006.

19. Masi G, Loupakis F, Salvatore L, Fornaro L, Cremolini C, Cupini S, Ciarlo A, Del Monte F, Cortesi E, Amoroso D, et al: Bevacizumab with FOLFOXIRI (irinotecan, oxaliplatin, fluorouracil and folinate) as first-line treatment for metastatic colorectal cancer: a phase 2 trial. Lancet Oncol 11: 845-852, 2010.

20. Park SB, Lin CS, Krishnan AV, Goldstein D, Friedlander ML and Kiernan MC: Oxaliplatin-induced neurotoxicity: Changes in axonal excitability precede development of neuropathy. Brain 132: 2712-2723, 2009. 